Meta

Journal des traducteurs

Translators' Journal

\title{
Étude des nouveaux concepts, des sigles et des emprunts dans la terminologie de la circulation routière et de leur ancrage dans la réglementation routière belge
}

\section{Linda Dewolf}

Volume 60, numéro 1, avril 2015

URI : https://id.erudit.org/iderudit/1032405ar

DOI : https://doi.org/10.7202/1032405ar

Aller au sommaire du numéro

Éditeur(s)

Les Presses de l’Université de Montréal

ISSN

0026-0452 (imprimé)

1492-1421 (numérique)

Découvrir la revue

Citer cet article

Dewolf, L. (2015). Étude des nouveaux concepts, des sigles et des emprunts dans la terminologie de la circulation routière et de leur ancrage dans la réglementation routière belge. Meta, 60(1), 173-192.

https://doi.org/10.7202/1032405ar

\section{Résumé de l'article}

La terminologie de la circulation routière se situe dans un environnement particulier lié au caractère varié et évolutif du langage technique, à de nouvelles pratiques policières, à de nouvelles règles en matière de circulation routière et à la place importante que tient le contexte juridique international. Tous ces facteurs contribuent à l'émergence de nouveaux termes posant des problèmes corollaires relatifs à la recherche d'équivalents et à l'absence d'harmonisation. Partant de ce constat, dans cette étude nous nous sommes fixé comme objectif d'exposer les mécanismes d'emprunts, de néologie et de siglaison. Les définitions des nouveaux concepts sont situées dans le cadre législatif belge du code de la route, de la loi de base et des arrêtés royaux d'exécution.

L'analyse de tout ce processus d'enrichissement terminologique aboutit à une procédure de validation interne décrite dans la méthodologie, qui vise à garantir la compatibilité du concept dans son environnement juridique et souligne l'importance d'impliquer les experts pour préciser les concepts techniques. Elle corrobore enfin l'hypothèse qui visait à établir une cote de fiabilité dépendant du degré d'ancrage de ces termes dans l'usage, le jargon policier et la législation belge.
Ce document est protégé par la loi sur le droit d'auteur. L'utilisation des services d'Érudit (y compris la reproduction) est assujettie à sa politique d'utilisation que vous pouvez consulter en ligne.

https://apropos.erudit.org/fr/usagers/politique-dutilisation/ 


\title{
TERMINOLOGIE
}

\section{Étude des nouveaux concepts, des sigles et des emprunts dans la terminologie de la circulation routière et de leur ancrage dans la réglementation routière belge}

\author{
LINDA DEWOLF \\ Vrije Universiteit Brussel, Bruxelles, Belgique \\ linda.dewolf@vub.ac.be
}

\section{RÉSUMÉ}

La terminologie de la circulation routière se situe dans un environnement particulier lié au caractère varié et évolutif du langage technique, à de nouvelles pratiques policières, à de nouvelles règles en matière de circulation routière et à la place importante que tient le contexte juridique international. Tous ces facteurs contribuent à l'émergence de nouveaux termes posant des problèmes corollaires relatifs à la recherche d'équivalents et à l'absence d'harmonisation. Partant de ce constat, dans cette étude nous nous sommes fixé comme objectif d'exposer les mécanismes d'emprunts, de néologie et de siglaison. Les définitions des nouveaux concepts sont situées dans le cadre législatif belge du code de la route, de la loi de base et des arrêtés royaux d'exécution.

L'analyse de tout ce processus d'enrichissement terminologique aboutit à une procédure de validation interne décrite dans la méthodologie, qui vise à garantir la compatibilité du concept dans son environnement juridique et souligne l'importance d'impliquer les experts pour préciser les concepts techniques. Elle corrobore enfin l'hypothèse qui visait à établir une cote de fiabilité dépendant du degré d'ancrage de ces termes dans l'usage, le jargon policier et la législation belge.

\section{ABSTRACT}

This study approaches terminology used in road traffic documents. It is demonstrated that this terminology shows variation which can be explained in different contexts related to the varied and evolving nature of technical language, new police practices, new traffic regulations and also to the important role played by international legislation. All these factors contribute to the emergence of new terms causing problems when looking for equivalents in several languages due to lack of harmonization. Based on this observation we have attempted to study the mechanisms of loans, neologisms and abbreviations. The definitions of the concepts are analysed in the Belgian legal framework of the Highway Code, the traffic laws and the implementing royal decrees.

The analysis of the terminological enrichment process finally leads to an internal validation procedure described in the methodology that aims to ensure compatibility of the concept in the legal environment and stresses the importance of involving experts to clarify technical concepts for translators. Finally, it supports our initial hypothesis that it is possible to establish a reliability status depending on how deeply these terms are rooted in usage, police jargon and Belgian legislation.

\section{MOTS-CLÉS/KEYWORDS}

circulation routière, réglementation, emprunts, néologie, contextes définitoires juridiques traffic, traffic laws, loans, neology, legal definitional contexts 


\section{Introduction}

Nouvelles réglementations, nouvelle classification des infractions, nouveau catalogue des amendes, nouvelles règles du Code de la route: au fil du temps, la législation relative à la circulation routière a connu de nombreuses modifications, d'autant que la problématique de la sécurité routière est une question d'intérêt national et un idéal sociétal recherché. Cette évolution, et en particulier la conjonction des facteurs que nous venons d'évoquer, explique que la terminologie se soit affinée en fonction des nouvelles infrastructures. Les avancées technologiques soulignent l'importance de l'emploi cohérent des termes nouveaux qu'il convient d'utiliser, que nous soyons ou non des experts. La circulation routière constitue un domaine susceptible d'être abordé sous de nombreux angles: du transport de personnes aux transports exceptionnels en passant par les véhicules agricoles. Tous font appel à de nombreux concepts spécifiques aux activités de la police fédérale de la route. Les agents verbalisateurs, comme les usagers de la route, sont confrontés à une technicité et à une complexité du langage administratif qui représente des obstacles majeurs à sa compréhension et mène souvent à des échanges improductifs et frustrants.

La présente étude s'inscrit dans la continuité de traductions effectuées au sein du Centre d'expertise et de connaissance en circulation routière de la police intégrée belge (Centrex). L'intérêt que nous portons à cette terminologie est double: linguistique et traductologique. Nous présenterons d'abord le corpus de travail, la démarche méthodologique suivie et le dépouillement terminologique. Ensuite, nous situerons les concepts dans leur champ sémantique. Nous nous attacherons à comprendre comment de nouveaux termes se forment dans un contexte spécialisé et tenterons d'expliquer les mécanismes de formation des néologismes et des sigles utilisés dans le cadre policier. Notre démarche tente d'établir une mise au point qui s'appuie sur une recherche théorique et sur notre expérience de traduction.

Notre approche a été guidée par le besoin de clarifier, de consolider et de systématiser un langage de spécialité susceptible d'être utilisé comme outil d'harmonisation terminologique. Par ailleurs, certaines abréviations ou certains termes ont, dans le langage usuel, une acception qui n'est pas celle employée dans le contexte de la circulation routière (c'est, par exemple, le cas du mot killer), raison pour laquelle il importe d'adopter des définitions précises, de lever l'ambiguïté et d'éviter tout flou notionnel. Une mise au point s'imposait enfin eu égard à l'importance et à la nécessité de clarté des messages et des communications, d'où l'attention accordée à l'usage des mots et de la langue par des spécialistes du domaine, le «real language usage» pour reprendre une expression que nous empruntons à Temmerman (2000: 31).

L'un des principaux enjeux de notre travail vise à proposer les termes et les expressions appropriés afin de pouvoir parler de leur évolution sans recourir de façon excessive à des termes étrangers et d'assurer la cohérence entre les traductions. Nous exprimons une certaine réserve face à la prolifération des anglicismes là où il existe un équivalent français, sauf si l'usage de l'anglais dans le cadre de la législation européenne, voire internationale, est adapté au besoin. Nous ne nous sommes pas contentée de définitions dictionnairiques, nous avons cherché à fournir un contexte ou des exemples dans le domaine de la circulation routière en excluant toutefois les définitions et explications de termes qui figurent dans des dictionnaires standard ou qui relèvent d'autres domaines tels que la signalisation routière, la prévention routière 
(domaine de l'Institut Belge pour la Sécurité Routière), l'assurance et l'accidentologie (par exemple, l'attestation de sinistralité) ou encore, l'infrastructure, l'aménagement et l'exploitation du réseau routier (compétence du Centre de recherches routières). En revanche, nous avons inclus dans cette étude les termes et les expressions relatifs à des matières gérées par des réseaux spécialisés comme l'Accord européen relatif au transport international des marchandises dangereuses par route (ADR), par exemple les termes et expressions arrimage, conduite sous l'influence de l'alcool ou de drogues, tachygraphe, transport de personnes, permis de conduire, véhicules agricoles. Il nous a également semblé utile, pour faciliter la communication, d'inclure une liste des abréviations employées dans cet environnement.

\section{Démarche méthodologique}

\subsection{Constitution $d u$ corpus}

Le corpus étudié présente, pour chacun des concepts relevés, un équivalent dans une ou plusieurs langues soit le français, le néerlandais, l'anglais ou l'allemand. Le travail de compilation entamé en 2011 recense les termes rencontrés au fil de nos propres traductions (5000 pages dans plus de 800 documents). Il s'agit de documents authentiques rédigés par des experts/juristes: notes, avis juridiques, brochures, rapports, ou d'autres textes à l'usage des policiers.

\subsection{Processus de dépouillement et de classification}

\subsubsection{Arbre notionnel}

La circulation routière est ventilée en de nombreuses matières, parmi lesquelles le transport de personnes, de marchandises dangereuses, le transport exceptionnel, les véhicules agricoles, les véhicules prioritaires, le transport de déchets, le transport d'organes... Le système de transport routier se compose de trois éléments qui interagissent entre eux, à savoir: la route et son environnement, la circulation et les véhicules qui en font partie, et enfin, tous les usagers de la voie publique.

Les différents domaines auxquels se rattachent les concepts étudiés sont regroupés sous une classification thématique (classification décimale [CDU] 656.05 en 656.01). L'organisation du domaine peut s'illustrer par un arbre notionnel. Dans cette arborescence, les liens représentent les relations sémantiques qu'entretient le terme «noyau» avec les descripteurs et les concepts subordonnés s'y rattachant. Le nœud central représente la tête de hiérarchie, le concept superordonné: wegverkeer - circulation routière. Cette structure pyramidale graphique permet de visualiser d'un coup d'œil le champ sémantique et les relations hiérarchiques et associatives entre les concepts génériques, spécifiques et associés.

$\mathrm{Si}$, dans certains cas, la possibilité d'attribuer à un terme plusieurs domaines pouvait être envisagée, nous nous sommes néanmoins limitée à indiquer un domaine préférentiel. Par exemple, les termes arrimage et tachygraphe disposent d'une terminologie technique complexe et parfois confidentielle en ce qu'elle aborde la problématique de la fraude. 


\subsubsection{Connaissance des domaines étudiés et contextualisation}

Consciente du fait qu'une telle étude exige des connaissances préalables de la matière, nous avons consulté les experts et les juristes ainsi que la littérature spécialisée. Hormis les études menées par Thomas Fallgatter (1988), Vlasta Křečková (1997), Leila Messaoudi (2002) et Elena Naujko (2005), rares sont, à notre connaissance, les travaux de recherche qui s'intéressent au développement des créations néologiques sous cet angle et dans ce domaine précis. L'expérience nous a montré à plusieurs reprises que la traduction d'amendements d'arrêtés requiert une approche pluridisciplinaire, tant du domaine technique que des textes juridiques comme le Code de la route, le Code de la rue, les conditions techniques, la réglementation ADR, les directives européennes, la Loi sur l'assurance responsabilité civile automobile (RCA ou WAM-wet - Wet Aansprakelijkheidsverzekering Motorrijtuigen en néerlandais), la Loi Salduz.

\subsubsection{Dépouillement terminologique}

Une consultation systématique des ouvrages de référence, dictionnaires et bases de données a été effectuée, notamment des documents de référence pertinents et accessibles sur Internet, des documents émanant d'organisations officielles ou d'organismes reconnus, tels que l'AIPCR/PIARC (Association mondiale de la route), l'ITRD (International Transport Research Documentation), la SWOV (Stichting Wetenschappelijk Onderzoek Verkeersveiligheid - Fondation néerlandaise pour l'étude scientifique de la sécurité routière), la VSV (Vlaamse Stichting Verkeerskunde - littéralement «Fondation flamande pour la science de la circulation routière»), la base de terminologie phraséologique de TISPOL et Euro Contrôle Route. Un aperçu des sources consultées lors des recherches terminologiques figure en annexe dans la section Références juridiques, qui répertorie les sources juridiques citées.

Les mots, ou expressions, traités sont classés par ordre alphabétique. Cette disposition, pratique pour l'utilisateur, présente l'inconvénient de mêler différentes catégories dans la même liste:

- des mots ou expressions relatifs aux véhicules (exemples: e-bike, trikes, oldtimer, side-car...);

- des mots ou expressions relevant des aspects techniques et dont le sens précis est connu des spécialistes (arrimage direct, arrimage couvrant ou de force, arrimage oblique, arrimage anti-rebonds, tests salivaires, tachygraphe, ADR, arrimage, cabotage...);

- des mots ou définitions relevant de la réglementation (rue cyclable).

\subsubsection{Elaboration des fiches}

Après avoir structuré la nomenclature en sous-ensembles thématiques pour l'étude et le traitement systématique des concepts, nous avons rédigé les entrées terminologiques proprement dites en appliquant la méthodologie de la recherche terminologique en vigueur selon les normes $\mathrm{ISO}^{1}$ et les Recommandations relatives à la terminologie $(1990)^{2}$. Nous avons créé une fiche pour chaque nouveau terme dont la signification nécessitait une définition ou pour tout concept existant devant être redéfini. Dans les définitions ou commentaires, les conventions utilisées sont: 
- Les mots-vedettes, c'est-à-dire les entrées du glossaire, sont en caractères gras.

- Les domaines d'emploi sont mentionnés entre chevrons; lorsqu'il y a un domaine et un sous-domaine, les deux éléments sont séparés par une barre oblique.

- Les équivalents sont précédés d'un code de langue. Les codes de langue utilisés s'inspirent de la norme ISO 639-1, Codes pour la représentation des noms de langue. Il s'agit de «en » pour l'anglais, de «fr» pour le français, « $\mathrm{nl}$ » pour le néerlandais et de «de» pour l'allemand.

Quant aux fiches, en voici trois exemples:

a) nl ademanalysetoestel <contrôles routiers >

Abréviation: AAT

Definitie: toestel waarmee het alcoholpercentage in het bloed wordt gemeten via de uitgeademde lucht. Dit toestel geeft een meetwaarde.

fr éthylomètre

Indicatif de grammaire: $n$. m.

Abréviation: ETM

en breath analyzer

Définition: l'analyseur d'haleine est un appareil qui mesure le pourcentage d'alcool dans le sang via l'air expiré.

Source juridique: Arrêtés royaux (A.R.) du 18 février 1913: d'une part, l'arrêté relatif aux appareils de test de l'haleine et d'autre part, l'arrêté relatif aux appareils d'analyse de l'haleine.

b) $\mathrm{nl}$ ademtesttoestel <contrôles routiers $>$

Abréviation: ATT

Definitie: toestel dat een indicatie geeft of een bestuurder mogelijk gedronken heeft. Op basis van deze indicatie wordt beslist of de bestuurder al dan niet een ademanalyse moet ondergaan. Dit toestel geeft geen meetwaarde.

fr appareil de test de l'haleine

Définition: appareil qui donne une indication du niveau d'imprégnation alcoolique d'un conducteur. C'est sur la base de cette indication que sera prise la décision de procéder ou non à une analyse d'haleine. Cet appareil n'effectue pas de mesure.

c) fr éthylotest < contrôles routiers >

Abréviation: ETT

Indicatif de grammaire: $\mathrm{n}$. m.

Définition: Appréciation du taux d'alcool dans l'air expiré au moyen d'un appareil; l'appareil lui-même.

Source: A.R. 18 février 1991 (voir note 3)

Le choix des entrées se justifie par leur fréquence en termes d'occurrence et par leur importance dans la réglementation routière. La rédaction des fiches terminologiques est guidée par le principe d'uninotionnalité suivant lequel chaque notion fait l'objet d'une entrée (terme vedette) dans la langue de départ suivi, le cas échéant, d'une définition, de notes conceptuelles et, enfin, de ses équivalents dans une ou deux autres langues (variantes utilisées aux Pays-Bas, en Suisse, en France ou au Canada). Les termes français et allemands sont suivis d'un indicatif de grammaire. Toutes les expressions qui apparaissent dans la nomenclature ne sont pas systématiquement accompagnées d'une définition, parfois c'est le contexte qui en explicite le sens. Certains renseignements, notamment les termes non retenus et les termes à éviter, figurent également sur les fiches. 


\subsubsection{Recherche d'équivalences}

Les difficultés rencontrées sont multiples, principalement en raison de la nature juridique du vocabulaire et des combinaisons de langues. Les équivalents proposés ne prétendent pas systématiquement assurer une parfaite symétrie terminologique ( Wechselzeichen»), mais tout a été mis en œuvre pour proposer une reformulation cohérente et proche dans la langue d'arrivée. Les solutions proposées et les choix effectués visent à garantir la compréhension de la notion. Pour ce qui est des équivalents anglais, nous avons consulté la terminologie disponible et diffusée sur Internet, notamment dans un document de référence de l'Association mondiale de la route (AIPCR). Ce type de recherche vise à répondre à un besoin précis et nous nous sommes demandé comment trouver l'équivalent de termes spécialisés. Pour trouver réponse à cette question, nous nous sommes fondée sur la définition d'équivalence parfaite que propose Dubuc:

Deux termes sont dits équivalents s'ils affichent une identité complète de sens et d'usage à l'intérieur d'un même domaine d'application. [...] Il y a équivalence même si chaque langue n'envisage pas la même notion sous le même angle. (Dubuc 2002: 55)

À défaut de définitions explicites, le recours à la signalétique apporte une aide conceptualisée à la recherche d'équivalents étrangers en s'appuyant sur la sémantique du message iconique.

Les exemples ci-dessous illustrent quelques-unes des difficultés de trouver les équivalents adéquats. Les signaux, par le langage international qu'ils véhiculent, permettent d'établir une distinction précise et immédiate des concepts auxquels ils se réfèrent.

Pour lever la confusion concernant les bandes bus et les couloirs bus, il faut faire la distinction entre le site franchissable qui ne fait pas partie de la voirie, et le couloir bus qui est un couloir privilégié sur la voirie. Il s'agit en pratique de deux concepts différents portant sur deux aspects législatifs différents du code de la route. Les panneaux de signalisation indiquent clairement que le site franchissable se trouve souvent au centre de la route, tandis que le couloir bus se trouve généralement sur le côté droit de la route.

Le couloir bus, en néerlandais busstrook, indiqué par le panneau de signalisation F17 de la figure 1, fait partie de la route. Il s'agit d'une bande réservée aux véhicules utilisés pour le service de transports en commun, de taxis et de véhicules affectés au transport scolaire.

FIGURE 1

Panneau F17

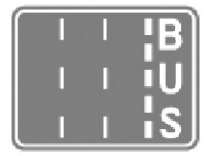

Le site franchissable (en néerlandais busbaan) qui ne fait pas partie de la route est indiqué par le panneau de signalisation F18 de la figure 2. Ce site n'est accessible qu'aux services réguliers de transports en commun. 
FIGURE 2

Panneau F18

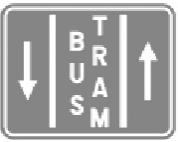

Autre distinction utile, celle entre marques d'immatriculation temporaire allemande et plaques temporaires françaises: les plaques temporaires (Kurzzeitkennzeichen) ne doivent pas être confondues avec les plaques de transit. Pour éviter toute confusion, les figures ci-dessous offrent un aperçu de ces deux types de plaques.

Plaque temporaire

Définition: marque d'immatriculation allemande temporaire de courte durée ou plaque à validité provisoire (de Kurzzeitkennzeichen)

FIGURE 3

Kurzzeitkennzeichen

\section{$\mathrm{K} \cdot 04060$}

Ce mot désigne des plaques d'immatriculation commençant par l'indicatif du canton (une à trois lettres), suivi d'un point, puis d'une série de chiffres débutant normalement par «03» ou «04». Elles sont aussi pourvues à leur extrémité droite d'une bande jaune avec une date de validité. Ces plaques d'immatriculation ne sont valables que sur le territoire allemand et disposent d'une durée de validité de cinq jours.

\section{Plaque de transit}

Définition: marque d'immatriculation temporaire allemande pour l'exportation (bande rouge)

$\mathrm{nl}$ : Duitse tijdelijke nummerplaat voor export (rode band)

FIGURE 4

Marque d'immatriculation temporaire allemande pour l'exportation

\section{MKK.458 C}

Ces plaques sont valables pour exporter en Belgique des véhicules venant d'Allemagne. Elles sont autorisées en Belgique à condition qu'à bord du véhicule se trouve un certificat d'immatriculation (Internationaler Zulassungsbescheinigung) sur lequel figurent le numéro de plaque, l'identité du titulaire et les données du véhicule sur lequel la plaque d'immatriculation est fixée. Le véhicule qui porte une plaque de ce type est enregistré dans la base de données officielle des autorités allemandes.

Des problèmes similaires se font également jour lorsqu'il s'agit de trouver la correspondance des infractions dans d'autres langues des États membres de l'UE. La correspondance ne peut être que linguistique étant donné que les concepts juridiques 
et les conditions d'une infraction diffèrent d'un pays à l'autre. À titre d'exemple, nous citerons les infractions mentionnées dans le code de la route belge sous les articles suivants, pour lesquelles nous avons cherché des équivalents à la définition en langue étrangère:

(1) Art.21.4.1. raccordements transversaux-dwarsverbindingen (nl), Querverbindungen (de), central reservations on motorways (en)

(2) Art. 22.1. Circulation sur les routes pour automobiles - verkeer op autowegen (nl) - Verkehr auf Kraftfahrstrasse (de) - traffic on dual carriageways (en)

(3) Art. 22septies 1.al.3. Accès aux rues réservées au jeu - verkeer in speelstraten (nl) - Verkehr in Spielstrassen (de) - play area - pedestrian priority (en)

Dans la signalisation routière, le signal C3 présenté à la figure 5 avec un panneau additionnel placé en dessous du disque apporte la précision nécessaire pour traduire ces concepts.

FIGURE 5

Panneau additionnel pour les rues réservées au jeu

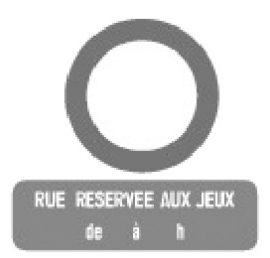

\subsubsection{Processus de validation - attribution d'un statut}

Nous avons émis l'hypothèse qu'il serait possible d'attribuer aux entrées du glossaire un statut correspondant à un niveau ou à une cote de fiabilité après les avoir soumises à un processus de validation rigoureux. Dans le scénario que nous proposons, chaque terme est soumis à un processus de validation à plusieurs niveaux et en diverses étapes.

\subsubsection{Choix des termes}

Nous avons recensé, au fur et à mesure de notre travail de traduction, les termes qui nous paraissaient spécifiques à un domaine et les avons inclus dans la liste des candidats termes en fonction de leur pertinence par rapport au domaine de la circulation routière.

\subsubsection{Collaboration avec l'expert}

La collaboration des experts s'avère, quant à elle, fondamentale pour l'établissement de la pertinence et de la justesse des termes tels que les concepts liés à l'arrimage (exemple: kopsjorren: arrimage anti-rebonds) et au tachygraphe.

À cette fin, il a été jugé pertinent d'y associer une démarche participative impliquant des experts de divers horizons, notamment des policiers qui contribuent quotidiennement sur différentes scènes à la sécurité routière et qui font partie des réseaux d'experts de la police fédérale de la route belge. 


\subsubsection{Relecture par un correcteur qualifié}

Première relecture linguistique: les «termes vedettes» sont relus par une tierce personne comprenant parfaitement la langue, qui procède à une première lecture pour s'assurer de la correction et du respect des règles linguistiques (syntaxe, orthographe, ponctuation et grammaire).

Deuxième lecture portant sur la clarté et la cohérence du contenu : l'objectif n'est pas de vérifier la validité (les caractéristiques spécifiques et stables) des informations juridiques, mais de contrôler que la rédaction de la fiche est parfaitement limpide et permet d'écarter toute confusion. Ce type de contrôle a été effectué, dans un nombre restreint de langues, par un correcteur ayant la connaissance du domaine.

La relecture-révision juridique vise enfin à assurer l'équivalence et le parallélisme quant au contenu juridique, selon le principe d'égalité des versions linguistiques français/néerlandais qui prévaut en Belgique.

Dans cette méthodologie, nous proposons aux relecteurs et aux experts des critères d'acceptation et de rejet des emprunts et des termes forgés. Ces critères sont fondés sur des paramètres linguistiques, mais aussi sur des paramètres extralinguistiques tels que l'acceptabilité et l'usage.

\subsubsection{Attribution d'un statut et d'une cote de fiabilité}

Chaque terme suggéré par le traducteur-terminologue donne lieu à une classification:

- terme pertinent ou non pertinent, avec proposition du spécialiste expert contacté;

- terme avec un commentaire (éventuellement «emploi critiqué»);

- terme proposé qui soit existe déjà dans une référence juridique, soit qui n’existe pas dans les références juridiques, ou terme à ajouter;

- terme préféré: terme qu'il convient d'utiliser, c'est-à-dire d'usage recommandé.

Les relecteurs sont invités à compléter les champs suivants pour chacun des termes candidats qui leur ont été soumis.

- Vérifié par: nom du relecteur ou de l'expert qui a procédé aux vérifications

- Révisé le: date de la relecture

- Non vérifié ou en cours de vérification

- Validé par: nom du relecteur ou de l'expert qui a procédé à la validation

- Non validé: terme non pertinent pour le domaine considéré

Le processus de validation proposé se termine par l'attribution d'un indice de fiabilité à chaque candidat terme selon une des qualifications suivantes:

- terme autorisé: terme «socialisé» ou conceptuellement acceptable, tiré d'une source traduite ou d'une source multilingue;

- recommandé par: s'il s'agit d'un standard;

- normalisé par: si le terme est officiel et d'usage obligatoire ou consacré par un texte de loi (dorénavant tous les termes publiés au Moniteur belge sont d'usage obligatoire dans la fonction publique) ou standardisé;

- proscrit, déconseillé, impropre ou rare: à éviter, par exemple, lorsqu'il s'agit d’un emprunt et qu'il existe une forme francisée officielle.

La principale difficulté que soulève la phase de validation tient essentiellement à la subjectivité des jugements. Nous avons donc posé comme principe que les termes proposés ont plus de chance d'être valides si les validations sont corrélées par plusieurs experts (validité interjuges). 
Dans son manuel de terminologie, Dubuc inventorie les procédés que la langue met à contribution pour désigner les réalités nouvelles et assurer la bonne intégration des néologismes. Cette démarche s'appuie, entre autres, sur l'exploitation de la documentation et la validation de celle-ci:

La qualité de la terminologie est en fonction de la valeur de la documentation qui la sous-tend. La validation des sources documentaires s'impose d'autant plus qu'Internet donne accès à des masses de documents dont la valeur est souvent fort aléatoire. (Dubuc 2002: 51)

Cette dernière démarche est bien sûr liée au public visé, mais dépend surtout de l'utilisation professionnelle de ce travail orienté vers une optique traductionnelle.

\section{Analyse terminologique - problématiques observées}

\section{1. Étude des néologismes}

Dans notre corpus, nous avons inclus une quantité importante de néologismes et nous avons cru pertinent d'étudier les combinaisons de termes constitués par dérivation ou autre formation syntagmatique. En français, les termes suivants sont formés soit par l'adjonction d'un affixe, soit par l'abrègement de l'un des deux ou des deux éléments du composé: $\boldsymbol{e}$-bike, écocombi... En néerlandais, les constructions linguistiques résultent de la combinaison de deux ou plusieurs termes-mots: blokrijden, file filteren (circuler entre les bandes de circulation et les files), frontaalflitsen (littéralement flash pris de face ou prendre en flash par l'avant et par l'arrière), klemrijden (bloquer la route au moyen d'un véhicule), spookvoertuig (véhicule fantôme), straatraces (courses de rue), politionele slachtofferbejegening (assistance policière aux victimes), verkeershandhaving (maintien préventif ou répressif de la circulation routière/contrôles routiers), vrachtwagensluis (sas pour camions), voorairbag (coussin de sécurité frontal), wielklem (sabot), weefstrook (voie d'entrecroisement), wegkapitein (capitaine de route).

Certains de ces termes ont été créés selon divers procédés de formation, sans que la liste soit exhaustive, nous pouvons entre autres mentionner:

a) la composition: ex. blokrijden, klemrijden, frontaalflitsen, verkeershandhaving, vrachtwagensluis;

b) les mots-valises: ex. : éco-combi, e-bike;

c) la combinaison de deux syntagmes nominaux : kwalificatiekaart bestuurder - carte de qualification du conducteur, cet exemple nous montre que si le néerlandais autorise la juxtaposition de divers éléments sans conjonction, l'équivalent français demande l'ajout de mots liens entre les différents substantifs.

\subsection{Néologie d'emprunt à l'anglais}

Nous avons repéré un certain nombre d'emprunts directs à l'anglais. Avant même qu'ils soient entérinés dans la législation, ces néologismes se sont intégrés dans l'usage, parce qu'ils répondent à une nécessité opérationnelle immédiate. Nous avons identifié des termes issus de l'anglais qui nous semblaient mal s'insérer dans la langue française dans la mesure où selon nous, leur forme est visiblement reconnaissable: camper, cruise control, dark number, enforcement, killers, stoopie, wheelie, ring, 
sampler, sampling, speedgun, trikes, truckruns, oldtimer, weigh in motion, etc. Beaucoup de termes ont des équivalents français ou néerlandais, mais ils ne sont pas toujours privilégiés par rapport au mot anglais, telles les expressions kiss and ride, kiss-and-go-zone, kiss-and-ride-zone; en français: zones de dépose-minute; en néerlandais: kiss-and-go-strook, kiss-and-ride-strook, zoen en vroem, kusje en weg ou aai en zwaai-zones. Nous constatons qu'il existe des variantes terminologiques aux Pays-Bas, la variante zoen en zoef- strook est très répandue. On retrouve également joyriding (balade dans une voiture volée), stunt (cascade), tuning (bolidage). Comme le montrent d'autres exemples ci-dessous, la sélection des équivalents français corrects suppose une très bonne maîtrise du domaine de spécialité.

Plusieurs mots et expressions utilisés tant par les policiers que par le grand public ont été empruntés ou sont des traductions littérales d'expressions anglaises. Citons le cruise control, correspondant américain du régulateur de vitesse, qui n'est pas l'expression adaptée, de même que le terme régulateur de croisière, qui est un calque (anglicisme syntaxique), est à éviter.
en: cruise control
$\mathrm{nl}$ : snelheidsbegrenzers
fr: régulateur de vitesse
Définition: système permettant à un véhicule de maintenir une vitesse constante, quelle que soit l'inclinaison de la route et sans intervention du conducteur.

Nous avons également relevé certaines variantes géographiques telles que oldtimer qui, selon le pays, donne lieu à des interprétations différentes. D’abord, précisons que pour désigner les véhicules dits ancêtres, oldtimer est un faux anglicisme (le terme anglais est "old car») utilisé comme terme générique pour les véhicules historiques. D'autres termes anglophones employés pour désigner des ancêtres automobiles sont: classic car, vintage car, et veteran car. De façon générale, la dénomination «voiture ancienne, oldtimer ou ancêtre» désigne les véhicules âgés de plus de 25 ans. Le pseudo-anglicisme youngtimer est également usité pour les véhicules anciens âgés de 15 à 30 ans. Dans l'usage linguistique allemand, l'expression oldtimer est en fait appliquée à tort aux véhicules anciens. Il désigne un véhicule automobile (en particulier une voiture, un camion, un autobus, une motocyclette ou un tracteur) âgé de 20, 25 ou 30 ans. En Suisse, les «Instructions concernant les véhicules vétérans » du 3 novembre 2008 de l'Office fédéral des routes définissent la notion de «véhicule vétéran». Dans le cas de truckrun il s'agit d'un emprunt direct pour lequel nous n'avons trouvé aucun équivalent en français ni en néerlandais. Par contre, le concept est défini dans la réglementation.

\section{en: truckruns}

Definitie: Truckruns zijn niet-commerciële parades voor charitatieve doeleinden waar vrachtwagenchauffeurs op geheel vrijwillige basis kinderen met een handicap in hun vrachtwagen meenemen voor een langzame rit door een stad of een aantal dorpen in België en hen zo een fantastische dag bezorgen. Deze activiteit heeft meestal tijdens het weekend plaats.

Définition: Les truckruns sont des parades à caractère non commercial et philanthropique au cours desquelles des conducteurs de poids lourds, sur une base volontaire, traversent à faible vitesse une ville ou plusieurs villages de Belgique, en transportant 
dans leur véhicule des enfants handicapés afin de leur offrir une journée exceptionnelle. Cette activité a généralement lieu durant le week-end.

Source: A.R. 19/7/2000

L'intégration de l'anglicisme killer dans le domaine de la sécurité routière nous a semblé particulièrement intéressante: killer se traduit habituellement par: principales causes de décès/de mortalité sur les routes, plaie/fléaux de nos autoroutes. Néanmoins, la recherche de l'origine de cette habitude dans un corpus de presse nous a permis de trouver un article intitulé Quatre tueurs traqués ${ }^{5}$ dans lequel le mot tueur désigne la «vitesse excessive», la «conduite sous influence», le «non-port de la ceinture» et le «non-respect des feux rouges». Le journaliste place «tueurs» entre guillemets pour mettre en relief qu'il l'utilise dans son sens premier originel.

Chacun des mots que nous venons de relever ici constitue un témoignage qui démontre l'influence d'une langue étrangère mise en contact par fertilisation.

\subsection{Les régionalismes et belgicismes}

L'étude de notre corpus met en lumière la présence, dans ce jargon spécifique, de belgicismes comme accident de roulage employé en Belgique pour traduire verkeersongeval. Le terme français officiellement recommandé est accident de la circulation. On peut trouver, sur le site d'un avocat ${ }^{6}$, un lexique juridique qui donne l'explication suivante:

- Accident de la circulation et accident de la route, au sens large, se disent indifféremment. Mais au sens strict, l'accident de la circulation se produit à l'intérieur des agglomérations, alors que l'accident de la route survient à l'extérieur (accident sur l'autoroute par exemple).

- Accident de la route et accident de la circulation se fondent dans l'expression accident de la circulation routière: "Dans le monde, les accidents de la route font chaque année plus de trois cent mille victimes.»

- L'accident de la circulation peut être matériel (dégâts uniquement matériels) ou corporel (personnes blessées ou tuées); dans ce dernier cas, l'accident est dit mortel.

Par analogie, l'élément qui dans infraction de roulage constitue un belgicisme ou plutôt un archaïsme, selon Kadlec (2005: 20), est roulage. Par conséquent, verkeersinbreuk se traduira de préférence par infraction au code de la route ou infraction routière.

Parfois l'étymologie des noms des véhicules peut être révélatrice d'une certaine proximité géographique malgré les variantes; c'est le cas de cuistax (BE) et rosalie (FR), ou d'un rapprochement lexical, comme entre cuistax et taxi.

La rosalie est une voiture à pédale ou quadricycle (parfois tricycle) dans le nord de la France. Le mot cuistax ou cuisse-tax, quant à lui, est un belgicisme.

Définition: véhicules comportant plus de deux roues (tricycles, quadricycles), munis d'un nombre variable de sièges et de pédaliers, loués aux estivants par des exploitants spécialisés en vue de la promenade ou du divertissement.

Etymologie: Le mot connu actuellement trouverait son origine dans l'expression "tasse-cuisse» ou "cuisses tassées», indiquant la contrainte exercée par ce cycle sur les cuisses. La dénomination flamande est billenblekker, littéralement «(engin) qui fait exhiber les cuisses». (Francard 2015: 130) 
Enfin, nous avons relevé quelques termes dont l'emploi est critiqué et pour lesquels certains dictionnaires recommandent un synonyme français. Le terme clignoteur (belgicisme) a un équivalent français: clignotant. Quant à airbag, il possède un équivalent français: sac gonflable ou coussin gonflable (plofkussen). Enfin, un crash test (à ne pas confondre avec crache-test le slogan d'une campagne de présentation du test salivaire au grand public) est un essai de choc en français, terme qui réfère à un élément d'étude de la sécurité issu du domaine de la biomécanique. Si en Belgique, la berme désigne l'espace qui sépare les deux chaussées d'une autoroute, ce concept porte, en français, le nom de terre-plein central.

\subsection{Noms propres - noms de marques}

Nous avons été particulièrement frappée par l'émergence d'une multitude de noms propres: Coyote, Dolly, Euro-codicil, Flexitank (citerne souple pour conteneur), Lidar (snelheidsmeter (cinémomètre en français), Provida, Rubalise, Segway, Springer (attache-chien pour vélo), etc. L'alcotest, variante orthographique de alcootest, est lui aussi une marque enregistrée de la société de droit allemand Drägerwerk AG \& Co. KgaA.

Comme le souligne Petit dans son étude, il convient de distinguer deux types de noms propres sur le plan référentiel:

les noms de sociétés [...] et les noms de produits ou de services [...], les produits ayant en général fait l'objet d'un brevet. Parfois les deux sont confondus, le nom de société désignant un produit, quand: le $\mathrm{N}$ de société est utilisé pour désigner le produit alors que celui-ci possède sa propre dénomination assurée par un lexème, par exemple: un Klaxon (avertisseur) [...]. Petit (2006: 692)

Et Petit de préciser que le nom de marque possède des propriétés juridiques : «En tant que marque, le nom de marque déposée (ND) doit faire l'objet d'un dépôt auprès d'un organisme officiel qui le validera s'il est conforme aux dispositions réglementaires et sera garant de sa protection juridique» (Petit 2006: 692).

Ces noms propres constituent des artefacts, comme l'anthroponyme Bob lexicalisé par antonomase. Le conducteur désigné, «celui qui conduit, c'est celui qui ne boit pas» s'appelle Capitaine de soirée ou Sam, en France (depuis 2005) ou encore Bob (depuis 1995) en Belgique (Bewust onbeschonken bestuurder, conducteur non alcoolisé conscient en néerlandais), Raoul au Luxembourg ou l'Ange en Suisse. Sam est également l'abréviation de «sans accident mortel». Considérant que le verbe bobber confère une dimension dépersonnalisée et renforce les effets du concept, l'Institut Belge de la Sécurité Routière a décidé récemment de redynamiser la campagne Bob en incitant les fêtards à penser dès le début de la soirée à s'organiser. D'un point de vue strictement linguistique, le verbe bobber n'existe ni dans le Petit Robert (2013), ni dans Le nouveau Littré (2005), même s'il y a fort à parier que le concept devrait s'implanter assez rapidement, du moins en Belgique. D'une part, on ne trouve point de traces de l'origine de ce superfétatoire dédoublement du B central, pourquoi bobber plutôt que bober, alors que la plupart des verbes courants se terminant en -ober ne prennent en effet qu'un «b» unique: dérober, englober, gober, lober, snober... D'autre part, il existe un risque de confusion avec le mot bobber qui désigne une motocyclette personnalisée dont on a enlevé les accessoires courants pour en réduire le poids dans un but d'esthétique minimaliste, un genre de «tuning» inversé. Le 
terme "gyropode» a été introduit par la Société française de terminologie et publié dans le Journal officiel du 26 mai 20097, afin de bannir l'utilisation du terme Segway, qui désigne le véhicule vendu par l'entreprise du même nom.

D'autres marques s'intéressent à cette technologie et se positionnent sur ce marché, via des concepts tels que le Winglet (gamme de gyropodes plus légers) qui pourrait voir le jour.

\section{Segway}

Définition: le Segway PT, ou Segway TP, pour Transporteur Personnel (anciennement Segway HT) est un gyropode, c'est-à-dire un véhicule électrique monoplace, constitué d'une plateforme munie de deux roues sur laquelle l'utilisateur se tient debout, d'un système de stabilisation gyroscopique et d'un manche de maintien et de conduite ${ }^{8}$.

Bien que le Segway soit couramment utilisé, notamment par les services de la police intégrée belge, le terme recommandé par la Société française de terminologie est gyropode.

Dans l'exemple suivant, nous remarquons deux orthographes différentes; rubalise est celle qui est la plus usuelle, bien que non déposée. Le nom composé de ruban et balise est un mot valise qui est également appelé «bande Ferrari» par les pompiers.

(4) Rubalise rue-balise

Définition: ruban servant principalement à la délimitation de zones, de chantier, ou au balisage de parcours.

Le rue-balise est utilisé par les secours publics pour interdire immédiatement l'accès à des zones d'accidents, délimiter des périmètres de sécurité ou canaliser des foules. La couleur dépend des autorités qui en font usage, autorité mentionnée sur ledit rue-balise?.

Nous attirons cependant l'attention sur le fait que la technique législative prescrite par le Conseil d'État de Belgique proscrit l'utilisation de néologismes pour lesquels, en l'absence d'alternative, il est conseillé de définir la notion.

\section{Formation des sigles et des acronymes dans les textes relatifs à la sécurité routière}

La siglaison a envahi l'environnement policier. Il s'agit d'un phénomène très présent dans le domaine de la circulation routière, en particulier lorsqu'il s'agit de désigner certains concepts liés aux statistiques et à l'accidentologie.

TABLEAU 1

Abréviations liées à l’accidentologie

\begin{tabular}{|l|l|}
\hline AccR & Accident de la route \\
\hline AccR DM & Accident de la route uniquement avec défauts matériels \\
\hline AccR LC & Accident de la route avec lésions corporelles \\
\hline
\end{tabular}

Bien que ces abréviations ne respectent pas les règles d'abrègement, notamment en ce qui a trait au point abréviatif, leur caractère officiel nous autorise à les orthographier tels que reproduits ci-dessus. Pourtant, si les abréviations les plus couramment employées: COL (Circulaire collective du Collège des Procureurs généraux 
auprès de la Cour d'appel - Collectieve Omzendbrief van het College van Procureursgeneraal bij de Hoven van Beroep), LPC (Loi Police de la Circulation), WPW (Wet Politieweg), etc., sont véhiculées dans le cadre policier, leur signification exacte n'est pas implicitement connue hors de ce contexte. En effet, certaines abréviations ont même plusieurs significations (voir plus loin ANPR). Les multiples abréviations policières ont fait émerger une forme de langage codé hermétique pour les non-initiés. La clé d'accès tient à toutes sortes de combinaisons d'initiales, de lettres, voire même de syllabes entières. Il est donc nécessaire de donner, en premier lieu, la signification complète, suivie de l'abréviation entre parenthèses et, par la suite, de réutiliser cette abréviation seule. On pourra ainsi écrire ou dire le Plan national de sécurité (PNS) et, ensuite, ne réutiliser que l'abréviation PNS. Selon Wolosin, des facteurs linguistiques et extralinguistiques expliquent la siglaison comme l'économie linguistique «promue par la réduction morphologique des termes techniques» (Wolosin 1996: 3) et l'économie rédactionnelle dans des notes. Le cryptage des termes techniques (et parfois non techniques) répond à une stratégie de simplification guidée par l'avantage d'économiser du temps à la rédaction et à la lecture de textes ou de messages, par exemple dans des ordres d'opérations.

Parmi les sigles dont l'occurrence a été recensée le plus souvent, nous avons, d'une part, identifié ceux qui représentent des noms propres, c'est-à-dire des noms d'organismes ou d'instituts comme l'AWV (Agentschap Wegen en Verkeer), la DIV (Direction d'immatriculation des véhicules) et l'IRU (International Road Transport Union - Union Internationale des Transports Routiers) ou d'associations comme la OVK-PEVR (Ouders van Verongelukte Kinderen - Parents d'Enfants Victimes de la Route) et, d'autre part, les acronymes.

TABLEAU 2

Acronymes

\begin{tabular}{|l|l|}
\hline KITAS & Kienzle Tachograph Sensor \\
\hline LIDAR & Light Detection and Ranging \\
\hline NAWAY & National Action on Highway \\
\hline
\end{tabular}

Les problèmes posés par la traduction des sigles et de leur(s) signifiant(s) sont multiples; nous rencontrons, entre autres, la difficulté de gestion de l'homonymie et de l'homographie: double siglaison ou pluriterminologie, dont voici plusieurs exemples:

(5) ANPR Automatic Number Plate Recognition ou Automatic license-plate recognition (ALPR); Automatic vehicle identification (AVI; Car plate recognition (CPR); License-plate recognition (LPR); Lecture Automatique de Plaques d'Immatriculation (LAPI)

Nous avons observé des variantes graphiques: sigles écrits avec ou sans points, en majuscules ou en minuscules, excepté la lettre initiale. Le tableau ci-dessous répertorie deux noms partageant un même sigle, ETM. Ce sigle fait référence, en France, à un: «radar mobile de nouvelle génération» qu'on appelle également ETM pour équipement de terrain mobile. En Belgique, cette abréviation est utilisée pour désigner un instrument de mesure de la concentration d'alcool dans les voies respiratoires. 
TABLEAU 3

Variantes homographiques

\begin{tabular}{|l|l|}
\hline ETM & éthylomètre \\
\hline ETM & équipement de terrain mobile \\
\hline
\end{tabular}

\section{L’ancrage juridique - contextes définitoires juridiques}

Dans la réglementation relative à la circulation routière, et particulièrement dans les arrêtés, la phraséologie est très normalisée et donc contraignante. Nous avons ainsi été amenée à nous interroger sur certaines impropriétés ou incertitudes terminologiques sources de confusion et à développer un processus de validation. Certaines entrées de notre glossaire abordent des difficultés de traduction: termes impropres, calques de l'anglais, lacunes terminologiques, etc. Ces problèmes peuvent, lors de la traduction, menacer la compréhension d'une note, d'un document, voire d'une loi. Nous croyons que pour parer à ces difficultés, il est utile d'identifier les termes anglais qui n'ont pas encore d'équivalent afin de créer des termes qui répondent aux besoins tout en respectant les normes du français. Depuis quelque temps, un appareil permettant de déterminer la présence d'alcool en prélevant un échantillon (sample) de l'air ambiant a fait son apparition sur le marché des accessoires destinés à la police. Ces nouveaux appareils sont plus sensibles que les appareils de mesure de l'haleine du fait de leur réglage: ils mesurent des concentrations d'alcool beaucoup plus basses, mais ne sont pas, à l'heure actuelle, conçus pour indiquer une valeur quantitative. En l'absence de nomenclature pour décrire ce nouveau dispositif, le terme anglais sampling a fait son apparition. Il s'agit d'une notion-clé que l'on pourrait évidemment paraphraser comme suit: "prétest/test indicatif/test sampling qui consiste à souffler dans un appareil qui indique s'il y a imprégnation alcoolique».

Nous trouvons dans la législation relative à la circulation routière (loi de base de $\left.1968^{10}\right)$, notamment dans le Code de la route et le Règlement technique, une série de définitions concernant les véhicules, les usagers (piétons) et les acteurs de la circulation (capitaine de route, coordinateur de la circulation, signaleurs). Toutefois, il semble assez surprenant que la notion de voie publique n'y soit pas précisée. À défaut de réponse dans le code, il faut se reporter à la jurisprudence pour chercher la définition de voie publique: "Constitue une voie publique, une voie qui est accessible à tous les usagers» (Cass. 20-11-1992 ${ }^{11}$ ). Par ailleurs, la loi elle-même exclut de son champ d'application certains véhicules tels que les pockets-bikes ou mini-motos.

Comme l'indique Cornu (1998: 265), la définition dans les textes juridiques se distingue de la définition terminologique en ceci: «la définition réelle se définit comme la détermination directe d'un élément de l'ordre juridique, la détermination, en droit, d'un concept». Les définitions légales sont les définitions qui apparaissent dans les textes de loi. Nous n'opérons pas ici, pour des raisons d'espace, les distinctions établies par Cornu (1998) entre définition réelle et définition terminologique au sein des définitions légales. Les définitions proposées par les lois et règlements ont pour objet de faciliter l'application de la loi en précisant le sens à donner aux mots et aux formules pour l'application de cette loi: ce sont des conventions interprétatives. 


\section{Nouveaux concepts ancrés dans la législation belge}

Les véhicules et les termes techniques décrivent des réalités et sont associés à des procédures spécifiques. Ils reçoivent des définitions précises dans les textes de loi ou les règlements auxquels nous faisons référence. Prenons un exemple de contexte définitoire juridique: le concept de «rue cyclable» a été introduit par la loi du 10 janvier 2012 parue le 3 février 2012 au Moniteur Belge en vue de son inscription dans le code de la route.

fr rue cyclable / nl fietsstraat

Définition: "2.61. "rue cyclable": une rue qui est aménagée comme une route cyclable, dans laquelle des règles de comportement spécifiques sont d'application à l'égard des cyclistes, mais dans laquelle les véhicules à moteur sont également autorisés. Une rue cyclable est signalée par un signal indiquant son début et un signal indiquant sa fin ${ }^{12}$."

Le passage en alternance des voitures en cas de rétrécissement routier ou manœuvre d'insertion, mieux connue sous l'appellation fermeture éclair, en néerlandais ritsen, est désormais explicitement prévu dans le Code de la route par l'Arrêté royal du 29 janvier $2014^{13}$, dans lequel a été inséré l'article suivant:

Art. 12bis. - Tirette.

Les conducteurs qui, lorsque la circulation est fortement ralentie, circulent sur une bande de circulation qui prend fin ou sur laquelle la circulation est interrompue, peuvent s'intercaler sur la bande libre adjacente seulement juste devant le rétrécissement.

Toutefois, lorsque pour traduire certains concepts en néerlandais, le français ne possède pas de terme simple, et que la réalité juridique évoquée existe, on peut avoir recours à une paraphrase explicative. Tel est le cas pour un nouveau concept de bandes de circulation ou «spitsstrook» qui a été introduit en Flandre en 2010 de manière à contribuer efficacement à la fluidité du trafic.

$\mathrm{nl}$ spitsstrook

Definitie: Een spitsstrook is een pechstrook die met signalering kan worden geopend als normale rijstrook.

fr: bande auxiliaire

Variantes: bandes de circulation ouvertes à la circulation pendant les heures de pointe, bande "heures de pointe», bandes d'arrêt d'urgence ouvertes à la circulation pendant les heures de pointe/voies supplémentaires de pointe ou voies auxiliaires dynamiques Équivalent en: peak lanes/rush hour lanes

Équivalent de: Sonderfahrtspur/Fahrspur in Spitzenzeiten

L'Arrêté royal du 13 février 2007 ${ }^{14}$ (Moniteur belge du 23/02/2007) introduit une nouvelle catégorie de véhicules: «les engins de déplacement». La législation reconnaît ainsi un statut juridique à cette catégorie de véhicules lents et modifie en outre la définition des «cycles».

(1) fr cycle: Ce terme désigne tout véhicule à deux roues ou plus, propulsé à l'aide de pédales ou de manivelles par un ou plusieurs de ses occupants et non pourvu d'un moteur, tel une bicyclette, un tricycle ou un quadricycle. L'adjonction d'un moteur électrique d'appoint d'une puissance maximale de $0,25 \mathrm{~kW}$, dont l'alimentation est réduite progressivement et finalement interrompue lorsque le véhicule atteint la vitesse de $25 \mathrm{~km} / \mathrm{h}$, ou plus tôt si le conducteur arrête de pédaler, ne modifie pas la classification de l'engin. 
(2) fr quads

Définition: sont considérés comme cyclomoteurs B à quatre roues, en tant que quadricycles à moteur ou en tant que véhicules lents, c'est-à-dire comme tracteurs agricoles ou forestiers ${ }^{15}$.

Autre définition: «Le quad, ou quadricycle selon l'appellation légale, est un véhicule non couvert motorisé tout terrain, monoplace ou biplace à quatre roues. Initialement à vocation utilitaire, il est de plus en plus employé comme véhicule tout terrain de $\operatorname{loisir}^{16}$.»

La publication de ces termes au Moniteur belge rend leur emploi obligatoire. Les définitions qui s'y rapportent sont dès lors officielles et recopiées in extenso du texte de loi.

\section{Conclusions}

Au terme de cette étude, une conclusion s'impose, même si les critères linguistiques sont importants pour l'intégration des termes dans notre glossaire. Nous espérons avoir démontré que des considérations techniques ou le contexte juridique entrent également en ligne de compte. Nous nous sommes efforcée, tout au long de notre travail, d'appliquer une méthodologie homogène clairement définie pour l'attestation de certains termes. La vérification des équivalents est réalisée dans la mesure où la «force probante» de l'équivalent a pu être établie par l'attribution d'un statut terminologique ou par une cote de fiabilité. Lorsque le terme a été publié au Moniteur Belge ou est tiré d'une source officielle, c'est-à-dire des textes législatifs nationaux (loi, Arrêtés royaux ou ministériels), le terme consacré est classé comme "vérifié» ou normé (la cote de fiabilité la plus élevée) et le terme officiel est d'usage obligatoire. Un terme largement répandu et reconnu par les réseaux spécialisés comme étant implanté est «accepté»: terme indiqué par l'expert contacté (fiabilité moyenne). Un néologisme recevra la cote de fiabilité la plus faible et sera classé comme terme «proposé » par l'auteur du document d'où provient le terme, ou suggéré par un traducteur ou un réviseur. Les termes déconseillés, incorrects ou obsolètes, ou les faux amis faisant l'objet d'erreurs fréquentes ont été fournis sur la même fiche que le(s) terme(s) correct(s) et signalés comme termes déconseillés (par exemple, l'anglicisme trafic routier). Ces critères ou indices fournissent autant de pistes qui s'ouvrent à une réflexion aussi porteuse de diversité que l'est le sujet de l'étude.

Nous avons montré par ailleurs qu'un protocole de validation clair et guidé par un principe de pertinence, avec un contrôle interjuges, permet d'atteindre une bonne stabilité de validation, garantie de la fiabilité des jugements experts.

Les difficultés auxquelles nous avons été confrontées résultent de facteurs liés à la complexité du domaine. Il est possible que les modifications ultérieures de la réglementation routière, comme le projet de nouveau code de la route; le poids des réformes administratives et l'application de la Loi Salduz aient pour effet de compliquer davantage la situation et donnent lieu à de nouveaux termes et à de nouvelles expressions. À cela s'ajoute l'internationalisation (accords bilatéraux, paquet routier) de certains concepts qui se traduit par une plus grande perméabilité de la langue aux anglicismes. Nous nous demandons simplement si les modifications proposées en matière de circulation routière auront pour effet de rendre la terminologie plus homogène ou de la diversifier. 
Bien qu'elle n'ait fait que lever un modeste voile sur un chantier de grande ampleur, cette étude nous a permis de dégager des pistes de recherche intéressantes pour le développement subséquent du glossaire. Les nouvelles définitions se présentent comme une proposition d'usage du terme dans le cadre bien précis de la circulation routière. Les données terminologiques collectées rendent compte de la pratique courante au sein de la police de la route, mais la portée du degré de stabilité de l'échantillon reste à vérifier dans un corpus encore plus vaste. Ce travail de recherche fait le constat et témoigne, par le nombre de nouveaux termes, du dynamisme du domaine de la circulation routière et nous conforte dans l'idée que le néologisme trouve sa juste place pour exprimer une innovation technique (nouveaux types de véhicules, nouveaux dispositifs de contrôle routier). Il pourrait s'avérer nécessaire d'en créer plusieurs autres dans les années à venir.

Le champ de cette étude couvre une réalité très étendue et très spécialisée qui se reflète non seulement dans l'importance du contenu technique des mots, mais aussi dans l'apport d'usages étrangers. La publication d'un premier jet du glossaire, en dépit de ses imperfections et de ses lacunes, poursuit l'intention d'amorcer un processus d'harmonisation en vue d'une terminologie commune plus uniforme, du moins dans une perspective nationale.

Nous prévoyons explorer une méthode d'extraction automatique permettant une gestion systématique et dynamique de la terminologie. À cette fin, nous envisageons de mettre en application des outils de veille terminologique servant de "crochet» pour formuler des requêtes permettant de capter l'apparition de nouveautés technologiques. Nous ne pouvions envisager d'étudier la totalité du vocabulaire, telle n'est pas notre ambition. Le glossaire est voué à être mis à jour et complété en permanence. Nous espérons que des ajouts successifs enrichiront le document, que des précisions, voire des modifications interviendront ou, a contrario, que certains mots ou concepts traités dans la première version se révèleront inutiles. Il va de soi que tout commentaire, toute critique et toute suggestion venant des utilisateurs seront pris en considération.

\section{NOTES}

1. Normes ISO 704 - travail terminologique - principes et méthodes/Normes ISO 1087 - travaux terminologiques.

2. Conférence des services de traduction des États de l'Europe occidentale, groupe de travail Terminologie et documentation, CST (1990). Recommandations relatives à la terminologie. Berne: Chancellerie de la Confédération suisse.

3. Arrêtés royaux du 18 février 1991 relatifs, l'un aux appareils de test et l'autre aux appareils d'analyse de l'haleine, abrogés par l'A.R. du 21 avril 2007.

4. Arrêté royal du 19 juillet 2000 relatif à la perception et à la consignation d'une somme lors de la constatation de certaines infractions en matière de transport par route.

5. http://www.lalibre.be/actu/international/la-journee-europeenne-51b8c630e4b0de6db9bdfd68, consulté le 30 juillet 2014.

6. http://www.avocat-lemeillat.com/lexique-juridique-avocat-paris/lexique-juridique-lemeillat. php ?glossaire=all, en ligne, consulté le 30 juillet 2014.

7. JORF n 0120 du 26 mai 2009 , page 8729 , texte $n^{\circ} 110$, consulté le 27 octobre 2013, $<$ http://dictionnaire.sensagent.com/gyropode/fr-fr/\#cite_note-lettre14-0 $>$.

8. http://www.ltt.auf.org/IMG/pdf/Lettre_14_de_la_Societe_francaise_de_terminologie_ automne_2009.pdf, consulté le 27 octobre 2013.

9. JENB Productions, 21 septembre 2011 marque déposée par l'entreprise Thiollier SAS.

10. Loi du 16 mars 1968 relative à la police de la circulation routière. [M.B. 27-03-1968]. 
11. Arrêté de la Cour de cassation du 20 novembre 1992.

12. Loi du 10 janvier 2012 modifiant l'arrêté royal du 1er décembre 1975 portant règlement général sur la police de la circulation routière et de l'usage de la voie publique, en vue d'inscrire la rue cyclable dans le code de la route, M.B. 3 février 2012.

13. Arrêté royal du 29 janvier 2014 modifiant l'arrêté royal du 15 mars 1968 portant règlement général sur les conditions techniques auxquelles doivent répondre les véhicules automobiles, leurs remorques, leurs éléments ainsi que les accessoires de sécurité, l'arrêté royal du $1^{\text {er }}$ décembre 1975 portant règlement général sur la police de la circulation routière et de l'usage de la voie publique et l'arrêté royal du 23 mars 1998 relatif au permis de conduire.

14. Arrêté royal du 13 février 2007 relatif aux engins de déplacement. [M.B., 23 février 2007].

15. Article $1 \$ 2,15$ de l'Arrêté royal du 15 mars 1968 portant règlement général sur les conditions techniques auxquelles doivent répondre les véhicules automobiles, leurs remorques, leurs éléments ainsi que les accessoires de sécurité (MB, 28 mars 1968).

16. http://www.techno-science.net/ ?onglet=glossaire\&definition=1594, consulté le 27 octobre 2013.

\section{RÉFÉRENCES}

Cornu, Gérard (1998): L'art du droit en quête de sagesse. Paris: PUF.

Dictionnaire technique routier de l'AICPR (2007).

Dubuc, Robert (2002): Manuel pratique de terminologie. Montréal: Linguatech.

Fallgatter, Thomas (1988): La terminologie de la circulation routière telle qu'elle se présente dans les textes législatifs de la Confédération et du Canton de Berne. Genève: Université de Genève, École de traduction et d'interprétation.

FrancARD, Michel (1993) : Entre Romania et Germania: la Belgique francophone. In: Didier DE RoBillard et Michel Beniamino. Le français dans l'espace francophone (1). Paris: Honoré Champion, 317-336.

KAdLEC, Jaromír (2005): Particularités lexicales du français de Belgique. Écho des études romanes. 1:15-26.

KŘEČKovÁ, Vlasta (1997): Les tendances de la néologie terminologique en français contemporain. Studia minora Facultatis philosophicae Universitatis Brunensis.

Le nouveau Littré (2005). Paris: Garnier.

Messaoudi, Leila (2002): Le technolecte et les ressources linguistiques. L'exemple du code de la route au Maroc. Langage et Société. 1(99):53-75.

Naujko, Elena (2005). Les emprunts français de la sous-langue «sécurité routière» dans la terminologie allemande. Revue du Gerflint. Consulté le 2 avril 2015, <http://gerflint.fr/Base/ Russie3/Russie3.html>.

Petit, Gérard (2006): Le nom de marque déposé: nom propre, nom commun et terme. Meta. 51(4):691-705.

Petit Robert (2013). Paris: Le Robert.

Temmerman, Rita (2000): Towards New Ways of Terminology Description. The Sociocognitive Approach. Amsterdam: John Benjamins.

Wolosin, Claudia (1996): Problèmes de traduction posés par la siglaison dans le domaine des nouvelles technologies de l'information et de la communication. ASp. 11-14:147-160. 